POLLACK PERIODICA

An International Journal for Engineering and Information Sciences

DOI: $10.1556 / 606.2018 .13 .1 .3$

Vol. 13, No. 1, pp. 33-44 (2018)

www.akademiai.com

\title{
OPEN IOT-BASED TELEMEDICINE HUB AND INFRASTRUCTURE
}

\author{
${ }^{1}$ István PÉNTEK, ${ }^{2}$ Ábel GARAI, ${ }^{3}$ Attila ADAMKÓ \\ Department of Information Technology, University of Debrecen \\ e-mail: ${ }^{1}$ pentek.istvan@inf.unideb.hu, ${ }^{2}$ garai.abel@inf.unideb.hu, \\ 3adamko.attila@inf.unideb.hu
}

Received 31 December 2016; accepted 25 September 2017

\begin{abstract}
In this paper, a unique healthcare solution is described that supports the even more effective operation of the hospital information systems. The main question is whether the emerging opportunities of the Internet of things devices can also be exploited in the industrial hospital information system landscape. This demonstrated research describes the most feasible way to integrate the Internet of things capability into hospital information production systems. The initial goal was the design and implementation of a single, unified telemedicine hub offering community-based solution for integrated medical systems. This solution allows the intercepted information to be collected and interpreted at community level. The designed and implemented system acts as a transmitter between the physician and patient. The software solution operates with sensor-based information collected from the individual. Emerging Internet of things devices and solutions open new horizons for today's health care systems. The presented and detailed system provides the ability to real-time health-monitoring and in-depth health analyzing through open application programming interfaces. The telemedicine hub system makes it easier to integrate the Internet of things capability into the operating health care systems.
\end{abstract}

Keywords: Telemedicine, Hub, Internet of things, Fast data, Big data, Sensors, Open application programming interfaces

\section{Introduction}

Nowadays, most people have a smart phone, one or more fitness equipments, or other devices tracking their fitness activity and collecting health-related data during their daily sports activity or just when the user is walking on the street. The recorded data is useful when the data owner gets sick or has a simple medical examination [1]. In 
these cases, the physician accesses the earlier created medical records from the medicalor hospital information system and accesses the recorded health-related data from global health- information system in order to make the appropriate decision. The latter is not an option today because there is no available global telemedicine system that can be accessed from the current hospital information systems. Sensor-based devices are producing significant volume of structured and unstructured health-related data every day [2]. Currently, most health-related systems are working as an island with unique data structure, undocumented interfaces and processes. This type of operation does not help to integrate modern health-related solutions into these systems. The governments and healthcare industry leaders are trying to regulate the healthcare business with standards and regulations. The vendors have to adopt these into their systems, but usually, the adaptation takes too long, and it is also very expensive. The medical and hospital information system vendors are adopting still very slowly Internet-of-things capabilities into their systems.

In this paper the so-called telemedicine hub system will be described that offers unique healthcare solution to exploit the opportunities offered by the Internet-of-things technology. The presented hub system is based on publicly available Application Programming Interfaces (API) endpoints and it supports the integration of the healthrelated tools into the existing hospital information systems. The presented system can collect community-level data and will assist the integration of the collected data into medical examination processes. The existing hospital information systems and other health-related systems can use the telemedicine hub system as an additional data source alongside the existing ones.

\section{Research background}

\subsection{Laws and regulations}

In the designed telemedicine hub system, the most important question is the different laws and regulations in different countries.

In the European Union (EU) there are stricter regulations for handling patients' information than in the United States (US). The European Union does not give so much opportunity to perform experiments or make healthcare statistics with real health-related data without data transformation. This transformation hides the sensitive or personal attributes and makes the transformed data impersonal. In the EU, the used medical records must be transformed before being transmitting to external systems, however, after data transformation, the medical records may loose important attributes like weight, sex, age, location or earlier disease. The United States has more permissive regulations than the other countries, and the researchers have to do less transformation before processing the medical records. This attitude is very important because the telemedicine hub system defines community-based data structure with large volume of uncertified and unstructured data transferred relying upon API. It means that the system collects and shares health-related information captured by uncertified sensors. The system supports physicians for examining and analyzing measurement values before 
making health-care decisions [3]. Furthermore, the system is able to receive, transform, process and store certified data also.

In 1996 the United States created the Health Insurance Portability and Accountability Act (HIPAA), and in the same year this act was signed by President Bill Clinton. This act is one of the most important basis of the current US healthcare business. This act provides data privacy and security provisions for safeguarding medical information. The act has five sections; the most important for us is the second, describing the national standards for processing electronic healthcare transactions. This section requires healthcare organizations to implement secure electronic access to the health-related data. The demonstrated system is compliant with this section of health insurance portability and accountability act, because it uses only secure communication channels, and it encrypts the communication.

Another important milestone is the health information technology for economic and clinical health act, signed by President Obama and coming into force in 2009. Its purpose was to stimulate the adaptation of Electronic Health Records (EHR). It contains 25 criteria, 15 core requirements and ten optional requirements. This act defines the electronically protected health information exchange terms and regulations. At that time, healthcare systems generally did not handle Protected Health Information (PHI). However, in present systems, security rules are in place to protect recorded health information.

\subsection{Telemedicine system}

Telemedicine is the use of information technology and telecommunication to provide healthcare services from distance. Telemedicine improves access to medical services that would often not be consistently available in distant communities [4], [5].

This telemedicine hub system was designed and implemented during a two-year research program. This telemedicine hub system is designed to be open towards thirdparty medical solutions. The goal was not to create a medical or hospital information system-extension, because the medical health records could be very complex and overregulated, therefore difficult to handle them effectively. Another goal was to enable community-level data-scanning into productive health care systems. The operation of the telemedicine hub system is based on international standards and is open towards integration with standard-based open application programming interfaces.

\subsection{IoT will affect the modern medical solutions}

The Internet of Things (IoT) is the collection of physical devices, vehicles, buildings or any other items with networking capability. An IoT device is equipped with electronics, software and sensors. Network connectivity enables these items to collect data from their environment and exchange data with other devices or a central processing unit [6]. The Internet of Things 'movement' started in 2012 when some manufacturers started to build sensor- based devices with network-capability. There are reports and statistics regarding the increase in the figures of the IoT devices projecting exponential growth [7]. 
The Internet-of-things will change the overall medical landscape, the medical examination workflows and medical systems, too. Physicians can analyze the collected data during a medical examination. The collected data is uncertified, but if the physicians handle it correctly, the medical results could be more accurate. Another way to use the IoT devices in the healthcare systems is real-time analysis. In this case, the patient wears one or more devices equipped with health-related sensors, and the device sends the collected data to the hospital information system as often as possible or realtime. From the transported data, the hospital information system is able to create analytics or can alert the physician if the user's equipment reported abnormal data [8].

Some hospitals already deployed smart beds. These are equipped with multiple sensors and have active Internet connection. Smart beds are subsets of the Internet-ofthings and useful only in hospital environments under controlled conditions. A smart bed is able to send patient-related information to the medical information system or able to send alert to the nurses when necessary [9]. The smart beds communicate with other systems or machines through network connection. The machine to machine communication is a very important feature to create real-time emergency notification systems inside a hospital. In this case, the smart beds can report abnormal measurements directly to a central machine alerting the designated person responsible for starting the necessary urgent intervention [10].

The other very promising opportunity is remote health monitoring; IoT devices can be applied for that. In the current medical systems, there are suitable devices equipped with sensors. Heart rate and blood sugar levels are often metered; the patient gets a device and wears all day long, or checks the blood sugar level with a certified equipment. After 24 hours of measurement, the physician exports the measurement results and can analyze the captured records. If the device is capable of global system for mobile communication, then it can send alerts, notifications or the measurement values through the mobile network [11], [12].

These opportunities combined with cloud computing will positively affect the modern medical solutions, and the health-related systems should integrate the IoT capabilities to make more accurate medical services [13]. This paper describes a working solution to integrate community-based telemedicine hub into these systems with cost efficient way.

\subsection{Medical infrastructures}

Today, the most applicable healthcare systems work with obsolete standards and data structures. In some cases the vendors created custom standards and did not follow the industry standards. Today's medical systems are working as separated island systems, and the health information exchange is an unresolved problem in these solutions now. Laws and regulations make more complex these systems and the new module developments are very expensive because vendors must follow the regulations. For security reasons, health-related systems use generally private API and closed subsystems. The IoT revolution is not seen on the current healthcare market. In order to develop a distributed, scalable and community-based IoT module into these systems is too risky and requires module based education to the users e.g. physicians or nurses [14], [15]. 
There are some systems in the United States that can exchange health related information, and the US laws and regulations are trying to support it, but even in the US there is no central healthcare system. The European Union has stricter rules for collecting and handling health-related information. US and EU do not allow open API and information sharing with non-healthcare licensed systems. They treat user information as inaccessible to third parties [16].

As it was mentioned earlier, the goal is not to adopt all of these rules. The laws and regulations will be more permissive in the future, and the IoT will be an integral part of the hospital information and healthcare systems. The implemented telemedicine hub system does not handle sensitive health-related records, but collects, transforms, processes and shares information collected from volunteers. In the future, more information could be useful to create more accurate services.

\subsection{Cloud-based open API}

Open application programming interface often referred to as a set of publicly available routines, protocols, and tools for building software applications. Publicly available application programming interface provides every developer with programmable access to the system resources. The API is a set of requirements that govern how an application can communicate and interact with another application. Public API also allows developers to access certain internal functions of the specified program. In the simplest terms, an application programming interface allows programs to interact with each other [17]. In most cases, open API endpoints are Representational State Transfer (REST or RESTful) web services. This provides a way of interoperability between network-capable systems. The hub uses public API to communicate with the other systems and with the health-related IoT devices. The telemedicine hub provides multiple communication protocols based on the most recent industry standards. The implemented hub supports multiple API standards, e.g. representational state transfer, Simple Object Access Protocol (SOAP), HyperText Transfer Protocol sockets (HTTP socket).

API based communication gives the possibility to interconnect the health-related systems with a common interface. In the telemedicine hub system the open API can be used to share the collected information with the data consumers [18]. The interconnected systems are able to exchange health related data or use the collected data to improve the health-related processes in the integrated system. A set of open application programming interface endpoints has been implemented to communicate with other integrated systems.

\section{Design of telemedicine hub infrastructure}

As it was described earlier, it was designed and implemented to collect healthrelated information from the community and to make it available for the health-related systems through open application programming interface endpoints. Therefore, connection should be established between the hub system and the integrated systems. Every country uses a special unique identifier for patients. This identifier could be a 
universal link between our telemedicine hub and the integrated health care systems. The system was not designed to substitute the hospital information systems or healthcare systems, but it could be a useful module in these systems to improve the healthcare processes and to make the results more accurate.

The telemedicine hub system has distributed application structure. In this structure, the hub is the server, and the Internet-of-things devices are the clients. Clients are the service requesters, and they use server resources. Clients can connect through multiple ways to the hub system.

The most supported way is the web socket-based connection. The web socket is a communication protocol that provides full-duplex communication channels over a single Transmission Control Protocol (TCP). It is a standardized protocol, and it is fully platform-independent. It was designed for web browsers and web servers, but it can be used by any client or server application. The transmission control-protocol uses port number 80. This port is open in most environments, and the firewalls do not block the communication. The Internet of things devices do not need full duplex connection; the server does not send any useful information to the clients. However, the clients are able to send the measurements real-time, which is similar to streams. The telemedicine hub is able to send an acknowledgement to the client if the measurements received properly; this is the acknowledgment justifying the full duplex connection.

The other way to send measurement data to the server is to use the hypertext transfer protocol. It works with web requests. In this case the clients are able to send SOAP request or to call an application programming interface endpoint. The default message structures are JavaScript Object Notation with Padding (JSONP) and eXtensible Markup Language (XML) for the client using API.

It is recommended to use the web socket-based communication because that supports real-time data transfer and generates less data traffic. It could be difficult to use if the Internet connection is breaking up unexpectedly. In this case, the web socket will not work properly, and the clients must recreate the connection after the Internet connection is up. The connection build-up process is very resource intensive. Therefore, clients should avoid too much connection rebuilding.

Token-based authentication method is implemented to validate and authenticate the clients and integrated systems. When the clients authenticate themselves for the first time the system requires unique health- related identifier, and after successful authentication, the server returns a long-term token. Clients can communicate with this token until its validity's expiration. If the token is expired then the client must renew it, or the server will refuse the requests by the clients. Telemedicine hub uses only tokenbased authentication to authorize clients and integrated health care systems. The second version of the Open Authentication protocol (OAuth) was implemented in the hub system. This technology is also used in global commercial software solutions (Facebook, LinkedIn, Google or Twitter). OAuth 2.0 provides specific authorization process for web applications, desktop applications and for mobile phones. The specification is developed by the Internet Engineering Task Force Open Authentication Work Group. The main framework was published in October 2012.

The implemented telemedicine hub system is gathering the information through the solutions described earlier. After the measurements or health-related records posted to the system, the subsystems are starting to process it. The transformed data could be 
transported to the integrated health care systems even in real-time. The integrated healthcare systems also have more options to reach the collected information. The easiest way to retrieve data from the telemedicine hub is calling a restful API endpoint. In this case, the supported message structures are JavaScript Object Notation with Padding (JSONP) and eXtensible Markup Language (XML). Clients and integrated healthcare systems use the same API layer in the telemedicine system. If the integrated healthcare system wants to get the measurement data real-time, then it must implement web socket-based solution.

Another way to implement real-time data-connection is when the integrated healthcare system starts a long polling web-request. Communication with HyperText Transfer Protocol (HTTP) long-polling request is a standardized way to request new information from a server. In this case, the server holds the request open until new health-related data is available. When the client receives new measurement value, it sends immediately another request to the telemedicine hub, and repeats it as long as it is needed.

A telemedicine environment produces large amount of data. The telemedicine industry counts on exponential data growth in the next few years. Based on the wide range of the medical examinations and these examinations' results it could be recognized that data structure is very variable. An examination result could be binary, image, text or a simple number. Considering the data structure and the amount of data the best solution is to store this information in a system that is capable of processing and saving more petabytes of unstructured data besides that the system processes the information almost real-time. It follows that the system has to be distributed and scalable with huge storage capacity [19]. Fig. 1 explains how the telemedicine hub system can be integrated into the health care system and how the infrastructure works.

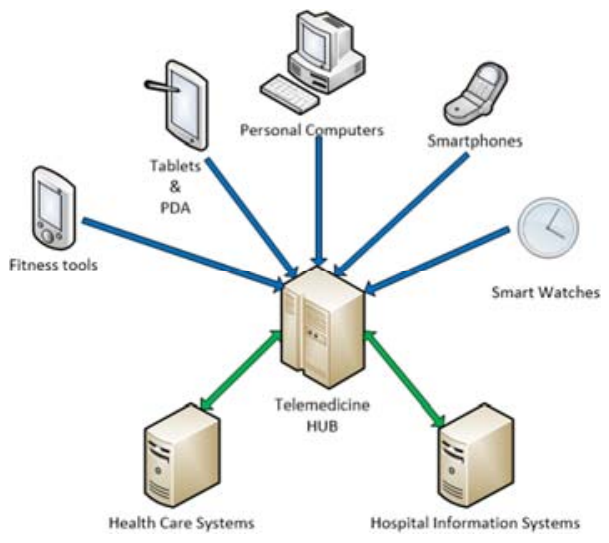

Fig. 1. Telemedicine hub infrastructure

The implemented telemedicine hub system has six separated modules, but there are dependencies between these modules. The implemented modules are the following (Fig. 2): 


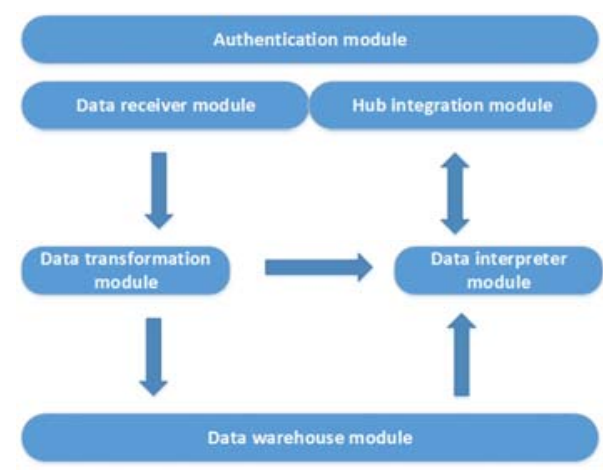

Fig. 2. The implemented modules

- Authentication module: this module is responsible for validating clients and integrated healthcare systems. The authentication module is the first in the application pipeline; it initializes the token-based communication. The clients and integrated systems can reach the other modules after successful authentication. This module is responsible for authorization, too. Clients and integrated systems are able to retrieve information if the user grants access to the personal health-related information. This module implements token-based authentication.

- Data receiver module: the IoT devices are using this module. The module is responsible for receiving health-related information and filtering the measurement errors and outliers. The first version of the system was trying to treat the outliers as an error, but the author's opinion is that an outlier measurement value could be a valid value, too. Outliers could be useful too, so the current version of the telemedicine hub system processes the outlier values and marks them with a specific flag. The data consumer's competence to decide how to handle the outlier values. This module uses k-mean classification to handle outliers and measurement errors. The receiver module works with WebSockets and HTTP requests, too. The client can choose which one is the most appropriate based on the quality of the actual network connection.

- Data transformation module: the receiver module transmits the incoming measurement values to the transformation module. This module transforms the large volume of unstructured incoming data in order to store it in the database. The data transformation is a resource intensive task; the implemented telemedicine hub system uses the in-memory operational database to store incoming data before the transformation. In-memory databases enable the implemented system to exploit the possibilities of fast data technology. The inmemory database solutions are fast but very memory intensive. The memory is the most expensive part of the server architecture, so this solution is more expensive than the general relational-database solutions. The advantage of the in-memory database solution is that the system does not require the received data to be stored on the hard disk before the transformation. In the telemedicine 
hub system, the in-memory database is a middleware solution and greatly speeds up the data processing. The data transformation module also provides the Health Level Seven International (HL7) standard messages to the integrated health care systems. The data transformation module works as a bi-directional model. The HL7 standard is the most popular standard to exchange health information between health-related systems [20]. The hub system supports version 2.3, 2.5 and 3 of the HL7 standard.

- Data interpreter module: this module is responsible for analyzing the transformed data and sends warning or alert if it was configured.

- Data warehouse module: after successful data transformation, the telemedicine hub system builds a data warehouse from the measurement values. This module works with data transformation module and frees space in the in-memory database. Later the system will support cognitive healthcare services that work with big datasets.

- Hub integration module: the module is responsible for communication with the integrated systems. This module implements a set of application programming interface endpoints. The API endpoints are publicly available. The integrated system can use this module via HTTP requests, or it can open a WebSocket to communicate with the telemedicine hub system. The authenticated clients can request transformed and interpreted data from the hub system, or it can subscribe to events, such as outlier blood pressure values. When an outlier value arrives the system sends a push request to the client requiring an opened WebSocket between the hub system and the integrated client.

A Windows universal application has been implemented to test the telemedicine hub system. The test application works with Microsoft Band smart watch. It supports the first and second versions, too. Microsoft band has heart-rate sensor capability and an easy-to-use programming interface. The implemented test application works with websocket protocol when the Internet connection is stable. If the device works in offline mode or under broken network connection, it collects the measurements and sends them to the telemedicine hub API in a batch when the connection is stable again. The application displays the actual heart rate value and sampling it in every second. Test client application is available in Microsoft Windows Store as a hidden application; it has been published in five versions until today. The client application is compatible with Windows 10 and Windows Phone 10 operating systems. Later a cross-platform application will be implemented to support the most wide-spread operating systems and to reach more users. With this cross-platform application, more types of smart watches and fitness tools will be supported. The current version of the implemented test client was created as a proof of the concept. Fig. 3 represents the current version of the Windows universal application while it is working with Microsoft Band.

The implemented telemedicine hub has an administration user interface where the user could follow the reported heart-rate values. It is a web-based user interface and fully browser-independent. It was implemented relying upon the HTTP long pollingrequest solution. The web browser sends a request to the telemedicine hub, and the hub holds the request until a new heart-rate value is received. When a new value is received, the server sends it to the client and closes the connection. The client sends a new long 
polling-request and repeats it. The web browser implementation refreshes a HyperText Markup Language (HTML) table and inserts the received value into the first line when a new heart-rate value is received. The web-browser client implementation illustrates healthcare system integration. Later the implemented system will be expanded with cognitive healthcare services and powerful web-based user interfaces. Now, this minimal user interface was enough to test the integration process. Fig. 4 represents the implemented web client while the telemedicine hub system is transmitting the received measurements.

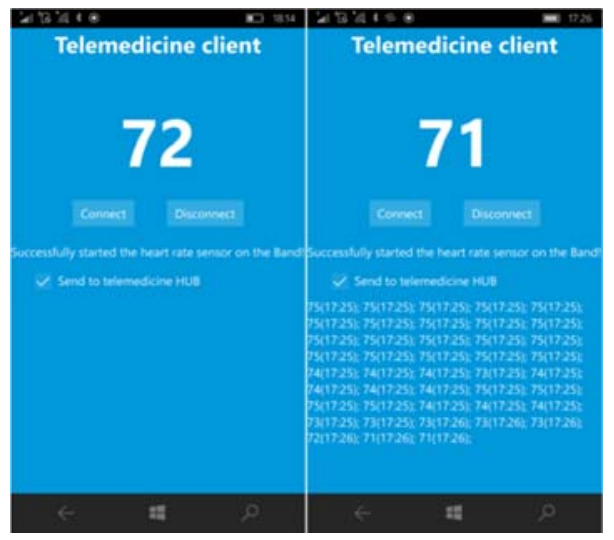

Fig. 3. The implemented test client

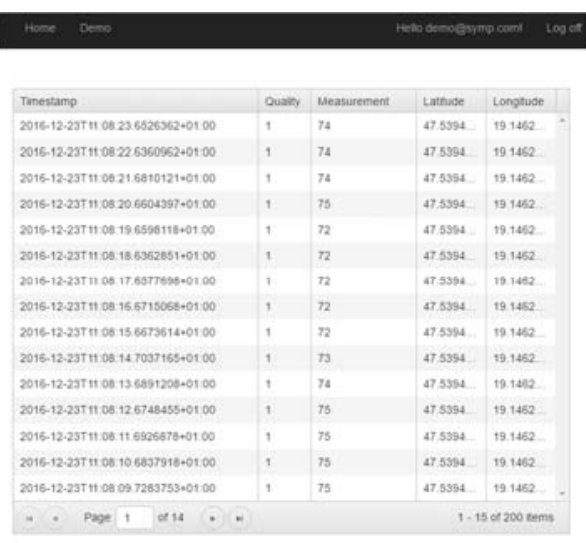

Fig. 4. The implemented web browser client

The implemented telemedicine hub system is a cloud-based distributed and highly scalable system. In the case of increasing workload, the system can be scaled up easily including new resources to the cluster. A load test was implemented in order to test the hub system's throughput. The load test has been started with five virtual machines with the following properties: 16 cores, 32 GB memory and 256 GB disk space. The 
implemented test was simulated in a working environment with clients and integrated health-related systems. The clients were producing the measurement values, and the hub system was transmitting the measurements to the simulated health-related systems. The number of simulated clients was increased until the maximum load of the counters of the cluster's resources was reached. The test was working with circa 100000 simulated clients and with 10000 simulated healthcare integrations. Clients were sending the test measurement values continuously to telemedicine hub system, at least one measurement value in every second. The hub system received, processed, transformed and sent the converted measurement values to the simulated healthcare systems. The test produced nearly 8500000000 simple heart-rate values during a twenty-four-hour test.

The number of opened network connection was the most critical factor during the test. Sometimes, the connected test devices lost the connections, and it resulted in more reconnection than expected earlier, but fortunately it did not cause any serious problem during the test.

\section{Conclusion and future works}

In this paper, one possible solution was presented to integrate the Internet-of-things capability into the existing health care systems and hospital information systems. The hospital information systems and other health-related systems should benefit from the IoT revolution. The hospital system vendors should implement this capability into their systems as soon as possible. A large volume of human health-related data is produced every day. The main question is that could the vendors use the opportunities given by the IoT devices? Open unified API could help the integration between the health-related systems and the presented telemedicine hub system. The main goal was to give an extension to the existing healthcare systems. In fact, the fitness trackers and other devices collect uncertified data, but the collected information could be useful during a medical examination. The healthcare industry evolves slower than the information technology. It is expected that the health-related systems will integrate the Internet-ofthings capability in the next ten years. It is conceivable that the vendors will use external services as data source. In the future, the authors will do more research to help to adopt the cutting-edge technologies into existing medical systems. Future explorations are recommended in connection with the open telemedicine interoperability hub's interconnections with other vendor's systems and with other manufacturer's wearable smart devices. The initial goal was to design and implement a unified telemedicine hub system that also provides a community-based solution to the medical systems where the integrated systems able to use the information collected within the community. The authors believe that the implemented telemedicine hub system and its architecture realizes the intended purposes.

\section{References}

[1] Estai M., Kanagasingam Y., Xiao D., Vignarajan J., Huang B., Estie K., Tennant M. A proof-of-concept evaluation of a cloud-based store-and-forward telemedicine app for 
screening for oral diseases, Journal of Telemedicine and Telecare, Vol. 22, No. 6, 2016, pp. 319-325.

[2] Kartsakli E., Antonopoulos A., Alonso L., Verikoukis C. A cloud-assisted random linear coding medium access control protocol for healthcare applications, Sensors, Special Issue on 'Sensors Data Fusion for Healthcare', 2014, pp. 9628-9668.

[3] Huang Y., Kammerdiner A. Reduction of service time variation in patient visit groups using decision tree method for an effective scheduling, International Journal of Healthcare Technology and Management, Vol. 14, No. 1-2, 2013, pp. 3-21.

[4] Bashshur R. L., Shannon G. W. History of telemedicine: Evolution, context, and transformation, New Rochelle, NY, Mary Ann Liebert, 2009.

[5] Fong B., Fong A. C. M., Li C. K. Telemedicine technologies: Information technologies in medicine and telehealth, Chichester, Wiley, 2011.

[6] Varshney U. Pervasive healthcare and wireless health monitoring, Mobile Networks and Applications, Vol. 12, No 2, June 2007, pp. 113-127.

[7] Luz M. P., de Matos Nogueira J. R., Cavalini L. T., Cook T. W. Providing full semantic interoperability for the fast healthcare interoperability resources schemas with resource description framework, International Conference on Healthcare Informatics (ICHI 2015), Dallas, 21-21 October 2015, pp. 463-466.

[8] Neelakantan P., Reddy A. R. M. Decentralized load balancing in distributed systems, Pollack Periodica, Vol. 9, No. 2, 2014, pp. 15-28.

[9] Kyriacou E., Panayides A., Constantinides P. mHealth eEmergency systems, in Telehealth and mobile health, Eren H., Webster J. G. (Eds), CRC Press, 2015, pp. 203-230.

[10] Varshney U. Pervasive healthcare and wireless health monitoring, Mobile Networks and Applications, Vol. 12, No. 2, 2007, pp. 113-127.

[11] Martinez L., Gomez C. Telemedicine in the 21st Century, Applied biostatistics for the health sciences, Nova Science Publishers, NY, 2008.

[12] Pindter-Medina J. mHealth and web applications, in Telehealth and mobile health, Eren $\mathrm{H}$., Webster J. G. (Eds.), CRC Press, 2015, pp. 395-415.

[13] Marciniak R. Role of new IT solutions in the future of shared service model. Pollack Periodica, Vol. 8, No. 2, 2013, pp. 187-194.

[14] Eren H., Webster J. G. The e-medicine, e-health, m-health, Telemedicine and Telehealth Handbook, Oakville, CRC Press, 2015.

[15] Poon C. Y., Hung K. F. mHealth: Intelligent closed-loop solutions for personalized healthcare, in Telehealth and mobile health, Eren H., Webster J. G. (Eds), CRC Press, 2015, pp. 145-160.

[16] ISO/IEC 7498-1, 1994 Information technology, Open systems interconnection, Basic reference model, The basic model (OSI-Model), International Organization for Standardization (ISO), Web, 6 June 2016.

[17] World development report, 2016, Digital trends, World Bank Group, IBRD (The World Bank), Washington DC, 2016.

[18] Adamkó A., Garai Á., Péntek I. Common open telemedicine hub and interface standard recommendation, The 10th Jubilee Conference of PhD Students in Computer Science, Szeged, Hungary, 27-29 June 2016, pp. 24-25.

[19] Garai Á., Péntek I. Adaptive services with cloud architecture for telemedicine, 6th IEEE Conference on Cognitive Infocommunications, Györ, Hungary, 19-21 October, 2015, pp. 369-374.

[20] Adenuga O. A., Kekwaletswe R. M., Coleman A. eHealth integration and interoperability issues: towards a solution through enterprise architecture, Health Information Science and Systems, Vol. 3, No. 1, 2015, pp. 1-8. 